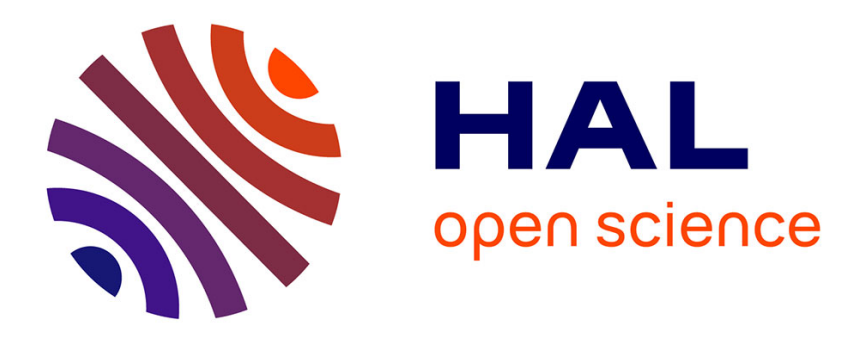

\title{
Can DERs fully participate in emerging local flexibility tenders?
}

Felipe Gonzalez, Marc Petit, Yannick Perez

\section{To cite this version:}

Felipe Gonzalez, Marc Petit, Yannick Perez. Can DERs fully participate in emerging local flexibility tenders?. 16th International Conference on the European Energy Market, Sep 2019, Ljubljana, Slovenia. hal-02283348

\section{HAL Id: hal-02283348 https://hal.science/hal-02283348}

Submitted on 10 Sep 2019

HAL is a multi-disciplinary open access archive for the deposit and dissemination of scientific research documents, whether they are published or not. The documents may come from teaching and research institutions in France or abroad, or from public or private research centers.
L'archive ouverte pluridisciplinaire HAL, est destinée au dépôt et à la diffusion de documents scientifiques de niveau recherche, publiés ou non, émanant des établissements d'enseignement et de recherche français ou étrangers, des laboratoires publics ou privés. 
archives-ouvertes

\section{Can DERs fully participate in emerging local flexibility tenders?}

Felipe Gonzalez, Marc Petit, Yannick Perez

\section{To cite this version:}

Felipe Gonzalez, Marc Petit, Yannick Perez. Can DERs fully participate in emerging local flexibility tenders?. 16th International Conference on the European Energy Market, Sep 2019, Ljubljana, Slovenia. hal-02283348

\section{HAL Id: hal-02283348 \\ https://hal.archives-ouvertes.fr/hal-02283348}

Submitted on 10 Sep 2019

HAL is a multi-disciplinary open access archive for the deposit and dissemination of scientific research documents, whether they are published or not. The documents may come from teaching and research institutions in France or abroad, or from public or private research centers.
L'archive ouverte pluridisciplinaire HAL, est destinée au dépôt et à la diffusion de documents scientifiques de niveau recherche, publiés ou non, émanant des établissements d'enseignement et de recherche français ou étrangers, des laboratoires publics ou privés. 


\section{Can DERs fully participate in emerging local flexibility tenders?}

\author{
Felipe Gonzalez Venegas and Marc Petit \\ GeePs - Group of electrical engineering - Paris \\ CNRS, CentraleSupélec, Univ. Paris-Sud, \\ Université Paris-Saclay, Sorbonne Université \\ Gif-sur-Yvette, France \\ felipe.gonzalezvenegas@centralesupelec.fr
}

\author{
Yannick Perez \\ Laboratoire de Génie Industriel \\ CentraleSupélec \\ Gif-sur-Yvette, France
}

\begin{abstract}
Distribution system operators' roles are evolving from a "fit-and-forget" approach towards an active management of their distribution grid. With this approach flexibility management at the local level can defer or avoid costly reinforcements and have a more efficient use of existing assets. Flexibility tenders are emerging as a mechanism that allow DSOs to procure flexibility for the medium to long term. This works proposed a modular framework to analyze the market rules of local flexibility tenders for the participation of distributed energy resources (DER), with a particular focus on demand response and electric vehicles, and applied it to the case study of UKPN's flexibility 2018/2019 tender and Enedis proposal for flexibility procurement. These tenders have been built to allow the participation of DER, but still there are barriers that may hinder their optimal participation, especially regarding connection procedures and baseline definition.
\end{abstract}

Index Terms-Demand-side management, Electric vehicles, Local Flexibility Markets, Vehicle-to-grid

\section{INTRODUCTION}

Distribution system operators (DSO) are facing a challenging environment with the massive integration of distributed renewable energy sources (DRES) like wind turbines and PV panels, and the electrification of new sectors, such as heating and transport. Ensuring grid reliability and quality of supply while integrating these new resources may require significant infrastructure investments by DSOs. However, advances in information and communication technology (ICT) and digitalization have enabled enhanced control and monitoring of distribution grids and flexibility assets, such as electric vehicles (EVs), stationary batteries and demand side response mechanisms (DSR).

In this context, DSOs roles and responsibilities are evolving. Historically, DSOs have operated their grids through a "fitand-forget" approach, since they did not require extensive management or monitoring. However, the integration of connected and smart flexible resources offers the opportunity for Active Network Management (ANM) of distribution grids,

This research benefits from the support of the Chair "PSA Peugeot Citroën Automobile: Hybrid Technologies and Economy of Electromobility", led by CentraleSupélec and ESSEC, and sponsored by Groupe PSA. changing the way distribution grids are operated and planned. The ANM approach can make efficient use of flexible resources at the local level, thus deferring or avoiding costly infrastructure reinforcements and helping the integration of renewable energy generation. Considering this, several use cases for flexibility can be considered [1]:

- Investment deferral: Flexibility can be used to reduce grid constraints (voltage or current issues), thus reducing the need for infrastructure reinforcements. This case requires a medium to long term vision of load (or generation) and flexibility availability for grid planning.

- Maintenance impact reduction: In this case flexibility is used to reduce the impact of asset maintenance in the distribution grid, which can be planned with several weeks in advance.

- Grid management in exceptional events: Flexibility is used to manage grid constraints in exceptional events, for example extreme weather conditions, an N-1 configuration or a grid incident. Flexibility would need to be procured close to real-time.

There has been an increasing interest from European regulators in the utilization of flexibility at distribution level. In particular, the Clean Energy Act [2] explicitly addresses the need for DSOs to procure flexibility. Also, the Council of European Energy Regulators (CEER), sees the future role of the DSO as a neutral market facilitator, ensuring a level playing field for all flexibility technologies. In this paradigm, DSOs ensure access to system-wide energy and flexibility markets to all actors and procure flexibility for the benefit of their grid when needed. To enable DSOs access flexibility, they foresee four main models:

- Rules-based: In this case flexibility is imposed by grid codes. For example, voltage regulation or reactive power compensation for distributed generation.

- Network tariffs: In this case tariffs provide the incentives to end users to alter their consumption patterns. However current network tariffs do not reflect the locational and temporal constraints of the distribution network.

- Connection agreements: DSOs and customers reach an agreement for the provision of of flexibility, like 
interruptible contracts for renewable generation.

- Market based: In this case, DSOs procure flexibility from market, either through bilateral contracts or a shortterm market platform.

This last model is preferred by regulators, where flexibility would be procured in a competitive manner [3]. In recent years research and several demonstrator projects have taken interest in market based solutions for flexibility procurement at the distribution level. Recent examples can be found in the Interflex project, where a flexibility market to solve intraday local congestion is being tested in two demonstrators in France and the Netherlands [4], and SmartNet, that tackles the TSODSO coordination problem, by proposing five market designs for flexibility procurement [5].

The mentioned demonstrator projects are based on shortterm markets. However, DSOs face high risks if they rely only on short-term markets to procure flexibility for investment deferral. Local markets may suffer from a lack of liquidity, thus exposing DSOs to high flexibility prices, or even endanger grid reliability, since they have limited options if the market fails. A solution to mitigate the availability and price risks is to procure flexibility through long-term agreements [6].

Within this context, UK Power Networks (UKPN), the London-area DNO, has adopted a "Flexibility first" approach towards all new investments in the high and medium voltage (HV and MV) grids [7]. For this, UKPN has implemented local flexibility tenders in order to contract flexibility for the medium to long-term (2019-2020 to 2023-2024 winter season, when peak load occur), in portions of the grid where they expect congestions. With this approach, UKPN can exploit costefficient flexibility solutions, thus reducing their investment costs. This tender process is the first market implementation of flexibility procurement at distribution level, to the authors best knowledge, and is expected to be followed by the rest of the DNOs in the UK.

In the present work, we analyzed distribution flexibility tenders with the objective of identifying barriers to entry for DER aggregators and propose possible evolutions of market design. The remaining of this work is organized as follows: Section II describes the modular framework for the analysis, Section III describes UKPN's local flexibility tender and a proposal launched by French DSO Enedis, and Section IV applies the framework to the tender proposal from the point of view of an EV aggregator. Finally, conclusions are made in Section V.

\section{MODULAR FRAMEWORK DEFINITION}

To analyze the market designs for the flexibility tenders, we adapted the modular framework proposed in [8], which was used to benchmark frequency regulation reserve markets in Europe for the participation of DER aggregators. The modularity of the framework allows to classify market rules and identify best practices and room for improvement, and can be adapted to emerging local flexibility markets.

This framework consists of three hierarchical modules:
A Administrative rules, barriers which can completely prevent the participation of DER into the market

B Product definition, which can limit the provision of flexibility by aggregators

C Payment Schemes, which can hinder the economic viability of the provision of flexibility services by aggregators

This framework was adapted to analyze DSO flexibility services, where DSOs procure active power flexibility to manage grid constraints and defer reinforcements.

\section{A. Module A: Administrative rules}

1) Technical discrimination: In the market design there might be rules impeding the participation of DER aggregators in the market, or requirements that would imply an excessive burden for their entrance to market. For example, the aggregation of multiple sites can be forbidden by a market rule, or connection or measurement requirements might not be adapted to aggregation of diffuse resources.

2) Inter-operability of flexibility platform: There is a wide variety of DSOs in European countries. For example, there is one major DSO in France, Enedis, which supplies 95\% of the territory, whereas there are more than 800 in Germany. Each tender will procure flexibility for one site, operated by one DSO only. However, as there could be a potential large number of tenders (and thus of DSOs), there should be a coherence and common process between these tenders. Aggregators, who could control assets across several DSO's sites, would benefit from these common structure, reducing their learning process.

\section{B. Module B: Product definition}

1) Distance to real-time and availability windows: This characteristic refers to how far ahead of delivery the flexibility products are procured. DSOs might need a long-term assurance of the flexibility availability when planning their grid, as relying only on a short-term markets might be prejudicial to them, but on the other hand aggregators of diffuse resources (such as demand response or EVs) might face high uncertainties on their asset availability ahead of time.

To reduce uncertainties for market participants, mid or long term procurement of flexibility may define a specific availability window in which the flexibility should be available for activation. Defining specific availability windows, (for example, corresponding to the expected peak-load hours instead of whole days), can help aggregators match their resources availability profiles to DSOs needs. It should be noted that even though flexibility should be available during these periods, they may not be (always) activated.

2) Activation time: This refers to the (minimum) amount of time the product (increase/decrease of production/consumption) is activated. This time will depend on the requirements of DSOs. However, long activation times might be highly constraining for aggregators of diffuse resources. For example, an EV aggregator would need to have a higher number of resources to sustain long activation periods, and may face higher operational constraints to ensure the mobility requirements of EV users. Also, since congestion management 
services are energy-based (instead of capacity-based services like frequency response), long activation times might induce increased battery degradation.

3) Minimum bid size: The minimum bid size will be linked to how many distributed resource units should be aggregated. Since local flexibility markets are focused on reduced areas, aggregating a high number units might be unfeasible. Low bid sizes will enable the participation of a higher number of flexibility resources and increase liquidity of the market.

\section{Location}

Local flexibility products are different from system-wide flexibility, such as frequency regulation, since the location of the resource is key in the impact it can have in solving a grid constraint. Products should have a well defined location, according to the expected grid constraints, to ensure that the flexibility resources will have an positive impact in grid operation.

\section{Module C: Verification and Payment}

1) Nature of Payments: For long-term flexibility procurement, both reservation (or availability) and utilization payments are needed. Reservation payments are necessary to ensure the availability of the flexible resources during the required availability windows. They are related to the flexible power capacity (in $€ / \mathrm{kW}$ for example). These payments may not be needed in close to real-time markets.

Activation payments refer to the actual utilization of flexibility, and are related to the energy provided during the flexibility service (thus, a payment is made in $€ / \mathrm{kWh}$ ).

Remuneration of flexibility for reservation and activation can be either market-based or a regulated tariff. A combination of market and regulated tariffs can be considered, such as a market-based reservation payment and a regulated tariff for the activation of flexibility. However, ideally both reservation and activation payments are based on declared bids, thus allowing to reflect different cost structures (fixed and variable) of flexibility resources.

2) Baseline definition and verification: Flexibility services imply a deviation from usual consumption/production patterns. Thus, it is necessary to determine a baseline upon which the activation of flexibility is verified. Adapted baseline methodologies should be used for diffuse resources, which may not respond to the same patterns as conventional, more predictable, resources. This issue has been solved at the transmission level, and services at DSO level should use this knowledge.

3) Stacking of services: Local flexibility services are not going to be the only source of revenues for DER aggregators. Participating in DSO services must not be exclusive with other flexibility (system-wide) markets, as long as they do not imply contradictory actions.

4) Environmental bonus: The development of flexibility services in the distribution level is closely related to energy transition and the better use of available resources. Thus, using clean technologies like renewable generation or storage should be preferred than appealing to fossil-fuel based resources, such as back-up generators.
TABLE I

MODULAR FRAMEWORK DESCRIPTION

\begin{tabular}{lll}
\hline Module & & Parameter \\
\hline Administrative rules & A1 & Technical discrimination \\
& A2 & Inter-operability of platform \\
Product definition & B1 & Distance from real-time \\
& B2 & Activation time \\
& B3 & Minimum bid size \\
& B4 & Location \\
Payment scheme & C1 & Nature of payment \\
& C2 & Baseline definition \\
& C3 & Stacking of services \\
& C4 & Extra Bonus \\
\hline
\end{tabular}

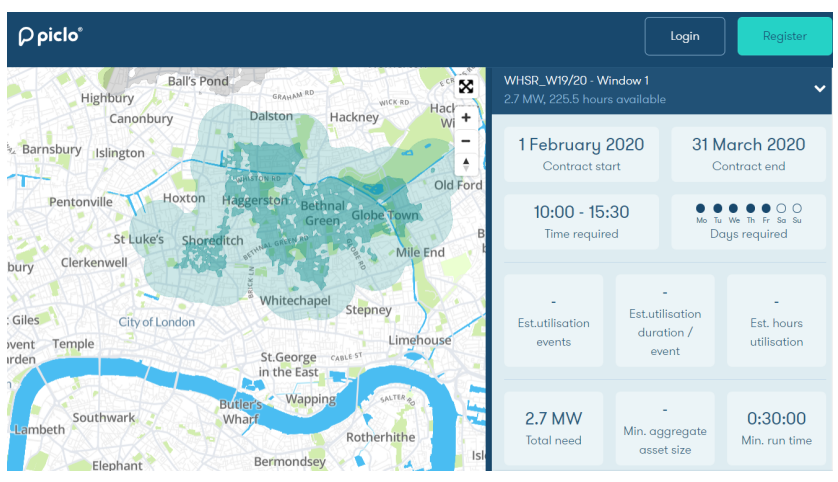

Fig. 1. Screenshot of Picloflex platform depicting a tendered site and the flexibility requirements

\section{EMERGING LOCAL FLEXIBILITY TENDERS}

\section{A. UK Power Networks}

In 2018, UKPN presented their Flexibility Roadmap, an ambitious plan to develop market-based solutions to procure flexibility for its network. The primary use of this flexibility is to defer load related reinforcements in their high and medium voltage network. For this, they set up a tender process to procure flexibility for identified sites in the grid where they foresee grid constraints, with delivery starting winter 20192020 (high load period). The tender process started in mid2018 with the publication of sites and a bidding period from December 2018 to March 2019. Results of the tender process were published in May 2019, contracting flexibility for 8 of the 27 tendered sites.

A platform, PicloFlex, was used for the tender, where flexibility sites and requirements where published and the market participants were able to declare their flexibility assets and submit their bids, as shown in Fig. 1. It is expected that the other DNOs in the UK will start their own flexibility tenders in the upcoming years, using the same platform.

Rules and product definition are described in [9]. The tender has been built with the possibility of DER participation. However, connection codes are strict, in particular for generation and storage assets (EREC G59 and EREC G83) and minutely measurements are required at the point of connection to the grid for each flexible unit. 


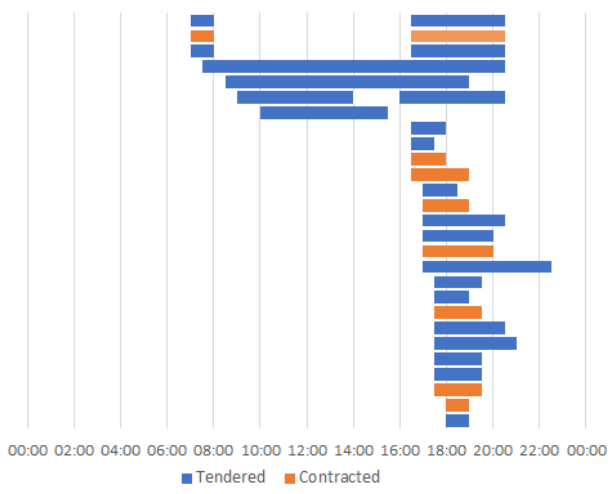

Fig. 2. Availability windows for the 27 tendered sites.

Regarding the products, each site required flexibility for a defined availability window of a few hours during the day (most of them during the evening peak time, as seen in Fig. 2), and for 2 to 5 months during winter. Flexibility outside the availability windows can be requested by the DSO, for constraint management in exceptional conditions, but flexibility providers are not obliged to accept them.

Minimum bid size was only $50 \mathrm{~kW}$, with overall flexibility requirements for each site ranging between 200 to 12600 $\mathrm{kW}$ for winter 2019-2020. Aggregation of different units is allowed, such as demand response, storage or distributed generation. Activation is made close to real time, with a minimum duration of activation of 30 minutes.

Considering payment and settlement, there is a reservation and an activation payment, both of which are set during the bidding process. Baselines, to which activation of flexibility are compared, are defined by the average half-hourly historical production/consumption during ten reference days defined by the DSO. This gives one fixed power value (not a profile) for the whole availability window and the aggregated flexibility assets. Alternative baselines can be used if suitable, which are based on recent-observation methodologies: for consumption sites is based on the average consumption of the previous five days during the availability window, and for storage or generation sites it is based on the average export in the half hour preceding the activation instruction.

Finally, stacking of services is allowed, as long as additional services do not interfere with the contracted services. Also, no environmental bonus was mentioned.

\section{B. Enedis (proposal)}

Since 2015, the article 199 of French Energy Law allows the utilization of flexibility for the optimization of distribution systems. Within this context, in 2018 Enedis launched a consultation process to set the bases for market-based flexibility procurement [1]. This document contained a proposal for a tender process, similar to the UKPN approach, where flexibility would be procured to avoid or defer grid reinforcements (foreseeable use of the flexibility) or to deal with exceptional events (unexpected use of flexibility) for their MV grid. The consultation period ended on February 2019 and a synthesis was published in June 2019 [10]. Since this is only a proposal of a market solution, actual characteristics of the process might be subjected to change.

The tender proposal stated a willingness to be open to all kinds of flexibility, allowing aggregation of different sites and stacking of services for flexibility providers. The process will be divided in three phases: first, a declaration of intention where Enedis will publish sites to identify potential flexibility providers. Second, a procurement phase, which can take the form of a tender if they see a sufficient number of market participants. Finally, the operational phase, where contracted flexibility is activated and the settlement and payments are made.

Flexibility will be procured for defined periods (availability windows) and with certain technical characteristics (minimum duration, number of activations, response time). However, these details are not yet fully defined. Similar to UKPN's case, flexibility could be procured outside the availability windows but market participants will not be obliged to provide flexibility.

The minimum bid size considered is $500 \mathrm{kVA}$. However, Enedis contemplates to accept only full bids (bids that can satisfy the whole flexibility needs for a given site). This imposes a de facto greater minimum bid threshold related to the grid flexibility requirement. Finally, the acceptance of bids will depend on both economical and technical characteristics of the offers. An environmental criteria based on $\mathrm{CO} 2$ emissions was considered at first, but then dismissed after the consultation period due to complex implementation.

Since flexibility will be procured ahead of time, capacity and activation payments are considered. While reservation payments will be subjected to competition, Enedis envisages to have regulated tariffs for activation payments according to the technology of the flexibility.

Finally, three baseline methodologies are proposed according to the flexibility assets characteristics: consumption or production sites, above or below a given power capacity threshold (36kVA).

- Recent observation: Used for production sites and adapted for controllable generators, the baseline is defined as the average power produced during a given time before activation, in this case the 10 minutes preceding it.

- k-nearest neighbors: Used for production or consumption sites, the baseline is defined as the average profile over $\mathrm{k}$ days during which the profile is the closest to the day of activation.

- Panel method: Used for consumption sites under 36 kVA (thus, adapted to diffuse demand response), this methodology sets the baseline by comparison to a reference panel of non-flexible customers with similar characteristics to the ones with demand response flexibility.

The proposed methodologies require more work from the DSO, like defining a metric for the closest days (k-nearest neighbors) and defining reliable panels of customers (panel method) but can provide baselines adapted to diffuse flexibility resources. 


\section{THE CASE FOR DIFFUSE DER: DSR AND EVS}

This section aims to identify the main barriers on market design for the participation of diffuse flexibility resources, such as demand response and electric vehicles. Table II resumes the criteria analyzed from the point of view of a diffuse flexibility aggregator.

Both initiatives state their willingness to allow participate of all kind of flexibility resources, allowing the aggregation of distributed flexibility. Also, they both encompass a sufficiently large area where these tenders will be applied, as the others DNOs in the UK will follow the same initiative, and Enedis encompasses over $90 \%$ of the French market. However, they can still present administrative barriers concerning connection and measurement. Pilot projects that use bidirectional capabilities of EVs (V2G technology) have found connection requirements too restrictive and time consuming, both in the UK and in France [11]. Also, UKPN's minute-by-minute measurement requirement do not align with standard smart meter technical capabilities, which usually have 30 minute time-resolution, thus making an extra barrier for demand-side flexibility. Easing connection process and aligning measurement requirements with standard smart meter capabilities should be considered to allow effective participation of all types of flexibility.

Considering the tenders, there is a conflict between the DSOs need of assurance of flexibility availability in the medium to long-term and the uncertainty of it by aggregators. Defining availability windows, ideally of no more than a few hours, can help aggregators match their availability profiles to DSO needs. Within this respect, UKPN's tender provide a good solution and can provide good opportunities for aggregators. For example, an EV aggregator can match the availability of company fleets that are parked after working hours to provide flexibility during evening windows.

Regarding the flexibility products, they should be adapted to distributed resources within a constrained geographical area (limited number of them). The main barrier detected is the minimum bid size considered in the Enedis proposal, where $500 \mathrm{kVA}$ and full bid constraint are not adapted to a local flexibility market. On the other hand activation times of up to 30 minutes are acceptable, and are compatible with standard smart meter measurements.

Finally, regarding the payment and verification, the main barrier detected are unadapted baselines methodologies, as in the case of UKPN's tender. Their baseline definition, based on the average power on reference days, present the benefit of simplicity and certainty of the base levels but it is not adapted to diffuse demand response. Demand profiles from controllable loads, such as water heaters, heat pumps or EVs, can vary widely between days due to weather conditions or usage patterns, and they can get blended with other uncontrollable loads present in the same buildings. Unadapted baselines methodologies can hinder the participation of these flexibility resources, since they might not receive full compensation for the activation of their flexibility. Enedis proposal provides baseline methodologies best suited to these flexibility resource.
TABLE II

ASSESSMENT OF THE CRITERIA FOR THE STUDIED TENDERS

\begin{tabular}{|c|cc|ccccc|cccc|}
\hline & A1 & A2 & B1 & B2 & B3 & B4 & C1 & C2 & C3 & C4 \\
\hline UKPN & +- & + & + & + & + & + & + & - & + & - \\
Enedis & +- & + & $?$ & + & - & + & +- & + & + & +- \\
\hline
\end{tabular}

\section{CONCLUSIONS}

Market-based solutions to exploit flexibility at the distribution level are starting to emerge beyond demonstrator projects, allowing DSOs to make an efficient use of their assets and thus defer or avoid grid reinforcements. These solutions need to be designed to be able to make use of all types of flexible resources. This work proposed a modular framework to analyze the market rules of local flexibility tenders for the participation of diffuse flexibility resources, such as demand response and electric vehicles, and applied it to the case study of UKPN's flexibility 2018/2019 tender and Enedis proposal for flexibility procurement.

These tenders were created to allow different types of flexibility assets to participate, but can still present some barriers. The long-term procurement can pose some challenges considering the need for certainty on flexibility potential for aggregators, which can be partly solved by proposing availability windows. Main barriers arise from restrictive and time consuming connection procedures, specially for storagebased flexibility resources such as EVs with bidirectional capabilities, and baseline methodologies that are not adapted to these kind of flexibility resources.

Finally, it should be mentioned that DSOs have allowed market participants to participate in the elaboration of these tenders, both in France and in the UK. Since these markets are just emerging, we expect that the feedback given by the first processes and the close collaboration with all stakeholders will allow an evolution of market rules for the benefit of all.

\section{REFERENCES}

[1] Enedis, "Les Flexibilites Locales Sur Le Reseau Public De Distribution D'Electricite," 2018.

[2] European Commission, "Proposal for a Regulation of the European Parliament and the Council on the internal market for electricity," Brussels, Tech. Rep. 5, 2016.

[3] Council of European Energy Regulators, "Flexibility Use at Distribution Level A CEER Conclusions Paper," Tech. Rep., 2018.

[4] Interflex Project, "D7.1 D7.2 District architecture requirements, tested innovations and use case planning," Tech. Rep., 2018.

[5] SmartNet Project, "Deliverable 1.3 Basic schemes for TSO-DSO coordination and ancillary services provision," 2016.

[6] USEF Foundation, "USEF: The Framework Explained," pp. 1-55, 2015 [Online]. Available: https://www.usef.energy/download-the-framework/

[7] UK Power Networks, "Future Smart: Flexibility Roadmap," 2018.

[8] O. Borne, K. Korte, Y. Perez, M. Petit, and A. Purkus, "Barriers to entry in frequency-regulation services markets: Review of the status quo and options for improvements," Renewable and Sustainable Energy Reviews, vol. 81, pp. 605-614, jan 2018.

[9] UK Power Networks, "Invitation to Tender v1.1," 2018.

[10] Enedis, "Les flexibilités locales sur le réseau public de distribution d'électricité. Synthèse de l'appel à contributions ouvert du 30 Novembre 2018 au 1er Mars 2019," 2019.

[11] Everoze and EVConsult, "V2G Global Roadtrip: Around the world in 50 projects," 2018. 